\title{
Downregulation of BCSG1 may correlate with better outcome of neoadjuvant chemotherapy for triple-negative breast cancer
}

\author{
JINSONG HE ${ }^{1}$, WEICAI CHEN ${ }^{1}$, HUISHENG WU ${ }^{1}$, MIN WANG ${ }^{1}$, YUAN WANG ${ }^{1}$, \\ XIAOJIA SHE ${ }^{1}$, SHUFEN SONG ${ }^{1}$, HONG GUAN $^{2}$ and XIANMING WANG ${ }^{1}$ \\ ${ }^{1}$ Center of Breast Diseases; ${ }^{2}$ Department of Pathology, Second Hospital of Shenzhen, \\ First Affiliated Hospital of Shenzhen University, Shenzhen, Guangdong 518035, P.R. China
}

Received May 2, 2012; Accepted September 4, 2012

DOI: $10.3892 / \mathrm{ol} .2012 .911$

\begin{abstract}
The aim of this study was to investigate the correlation between breast cancer-specific gene 1 (BCSG1) and the effect of neoadjuvant chemotherapy (NAC) in patients with triple-negative breast cancer (TNBC). Real-time RT-PCR and immunohistochemistry were used to determine the expression of BCSG1 mRNA and protein levels of 32 TNBC patients before and after NAC. Tumor size was reduced significantly after NAC in all 32 TNBC patients. The expression of BCSG1 was also decreased after NAC at both mRNA and protein levels. There was a negative correlation between BCSG1 levels after NAC and the effect of NAC. BCSG1 may be a potential target for NAC in the treatment of TNBC.
\end{abstract}

\section{Introduction}

Triple-negative breast cancer (TNBC) is a type of high-risk breast cancer in which the estrogen receptor (ER), progesterone receptor (PR) and human epidermal growth factor receptor (HER2/ErbB2) are all negative (1). TNBC accounts for $15-20 \%$ of all breast cancer cases. Since the effective targeted endocrine therapy cannot be used, patients with TNBC usually have a poor prognosis. In addition to surgical therapy, the main treatment for TNBC is chemotherapy. Neoadjuvant chemotherapy (NAC) has been widely accepted in the treatment of breast cancer. Despite the relative chemosensitivity, less than $25 \%$ of all patients with TNBC treated with standard NAC achieve complete pathological response (pCR). However, the evaluation of the effect of NAC is limited to the clinical and pathological changes of the tumors and metastatic

Correspondence to: Professor Xianming Wang, Center of Breast Diseases, Second Hospital of Shenzhen, 3002 West Sungang Road, Futian, Shenzhen, Guangdong 518035, P.R. China

E-mail: jinsonghe0114@126.com

Key words: SNCG protein, neoadjuvant therapy, adjuvant chemotherapy, breast neoplasms lymph nodes. Therefore, a method to simply and accurately evaluate the effect of NAC in the treatment of TNBC would be extremely valuable.

A breast cancer-specific gene, BCSG1, was identified by Ji et al (2) in 1997. BCSG1 is highly expressed in humaninfiltrating breast carcinomas but not expressed in normal or benign breast tissues and the expression of BCSG1 is also stage-specific for breast cancer (3). Overexpression of BCSG1 in breast cancer cells increases the motility and invasiveness in vitro and stimulates metastasis in vivo (4). In a clinical trial, patients with BCSG1-positive breast tumors generally had shorter disease-free survival and overall survival and higher probability of mortality compared with the patients with BCSG1-negative tumors (5). Therefore, BCSG1 may be used as a marker for breast cancer progression and prognosis $(5,6)$. Although a number of studies have established the significance of BCSG1 in breast cancer, few results concerning the correlation between BCSG1 and TNBC have been reported (7). Therefore, we analyzed the correlation between BCSG1 expression and the effect of NAC in the treatment of TNBC in the present study to determine the role of BCSG1 in the treatment of TNBC with NAC.

\section{Patients and methods}

Patients and treatment. All 32 patients (female, 27-45 years old; median age, 40) were treated at the Center of Breast Diseases at the Second People's Hospital of Shenzhen between September 2009 and August 2011. All patients were diagnosed with triple-negative invasive non-specific cancer by pathological evaluation and hormone receptors test. Patients underwent breasts, double axillary and liver type-B ultrasonic scan, chest X-ray and whole body bone scan prior to chemotherapy. The TNM stages were: IIA, 11; IIB, 14; IIIA, 5; IIIB, 2 (UICC/AJCC, 2003). A total of 18 patients were found to have ipsilateral axillary lymph node metastasis. No other treatment was administered prior to definite diagnosis. No serious heart, liver or kidney damage was detected (Karnofsky score $=100$ ).

All the 32 patients were administered a combination of $600 \mathrm{mg} / \mathrm{m}^{2}$ cyclophosphamide (CTX), $80 \mathrm{mg} / \mathrm{m}^{2}$ epirubicin 
(EPI) and $500 \mathrm{mg} / \mathrm{m}^{2}$ fluorouracil (5-FU) on day 1 and then every 21 days. After 2 cycles of chemotherapy, patients underwent modified radical mastectomy or breast-conserving surgery. The changes of lesions were evaluated according to the criteria of the World Health Organization (WHO) for anticancer drugs prior to and following NAC-based clinical response and $\mathrm{B}$ ultrasound (3). Complete response (CR) and partial response (PR) were calculated as clinical overall response. Breast cancer tissues were obtained through coreneedle biopsy prior to NAC or surgery following NAC. Written informed consent was obtained from the patients. The study was approved by the ethics committee of the First Affiliated Hospital of Shenzhen University, Shenzhen, Guangdong, China.

Immunohistochemistry. Immunohistochemistry was performed according to the manufacturer's instructions with BCSG1 polyclonal antibody (Santa Cruz Biotechnology, Inc., Santa Cruz, CA, USA; 1:100 dilution). Brain tissue was used as a positive control and PBS as a negative control. BCSG1-positive cells were defined according to the standard reported by Mohsin et al (8). The BCSG1 expression score was calculated from the proportion of positive cells and the color intensity of the cells: i) number of positive cells, grade 0 , $<25 \%$; grade 1, 26-50\%; grade 2, 51-75\%; grade 3, $\geq 75 \%$; ii) color intensity, grade 1, weak; grade 2, moderate; grade 3 , strong. The score for each slice is the summation of the two parts. A total score $<3$ was considered to indicate low expression and $\geq 3$ to indicate high expression.

Real-time RT-PCR. Total RNA was isolated from breast cancer tissue with TRIzol reagent (Invitrogen, Carlsbad, CA, USA) according to the manufacturer's instructions. The concentration and purity of total RNA were determined by a spectrophotometer (Eppendorf, Hamburg, Germany). Reverse transcription was performed using Random primer RT mixtures with M-MLV (20 U/ $\mu$ l; Promega, Madison, WI, USA) reverse transcriptase $20 \mathrm{U} / 20 \mu$, total RNA $1 \mu \mathrm{g} / 20 \mu \mathrm{l}$. cDNA was stored at $-20^{\circ} \mathrm{C}$. Quantitative real-time PCR was performed with an ABI stepone plus Real-time PCR system. The sequences of the primers were as follows: BCSG1, forward 5'-AGGAGGGGGTCATGTATGTG-3', reverse 5'-TTCTCTTTGGATGCCTCACC-3'; GAPDH forward 5'-GGAAGGTGAAGGTCGGAGT-3', reverse 5'-CCTGGAAGATGGTGAGGG-3'. PCR mixtures contained $1 \mu \mathrm{l}$ cDNA, $12.5 \mu \mathrm{l} \mathrm{SYBR}{ }^{\circledR}$ Premix 2X (Toyobo, Osaka, Japan) and $0.16 \mu \mathrm{mol} / 1$ forward and reverse primers in a total volume of $25 \mu \mathrm{l}$. Reactions were started with a polymerase activation step at $94^{\circ} \mathrm{C}$ for 5 min followed by 35 cycles of $94^{\circ} \mathrm{C}$ for $30 \mathrm{sec}, 57^{\circ} \mathrm{C}$ for $45 \mathrm{sec}$ and $72^{\circ} \mathrm{C}$ for $30 \mathrm{sec}$. Fluorescence data were acquired after each cycle. The amount of specific mRNA in samples was calculated using the $\Delta \Delta \mathrm{CT}$ method.

Statistical analysis. Measurement data are expressed as mean \pm SD. Data were analyzed using SPSS 13.0 for Windows (SPSS Inc., Chicago, IL, USA). The paired Student's t-test was used for comparing the BCSG1 expression difference before and after NAC. The $\chi^{2}$ test was used for comparing the clinical overall response rate in patients with high or low BCSG1

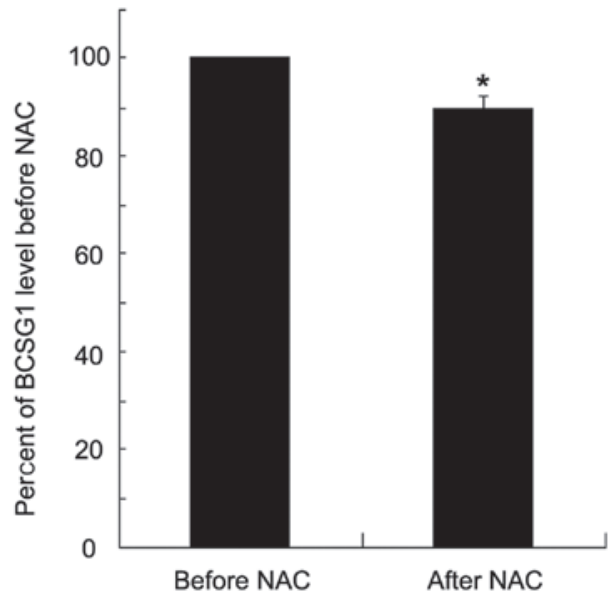

Figure 1. Expression of BCSG1 mRNA in the breast cancer tissues. Before and after NAC, breast cancer tissues were obtained through core-needle biopsy or surgery, respectively. The BCSG1 mRNA levels were determined by real-time RT-PCR. The amounts of specific mRNA in samples were calculated using the $\Delta \Delta C T$ method. Data are expressed as mean $\pm S D(n=32)$ ${ }^{*} \mathrm{P}<0.05$, compared with before NAC. BCSG1, breast cancer-specific gene 1; NAC, neoadjuvant chemotherapy.

expression. The correlation between the effect of NAC and BCSG1 expression was measured by Spearman rank correlation analysis. $\mathrm{P}<0.05$ was considered to indicate a statistically significant result.

\section{Results}

Response to neoadjuvant chemotherapy. The tumor softened in $62.5 \%$ of patients (20/32) 10 days after NAC and reduced in size in $71.9 \%$ of patients (23/32) after one cycle of NAC. Tumor size shrank markedly in $84.4 \%$ (27/32) patients after 2 cycles of NAC. There were $3 \mathrm{CR}, 24 \mathrm{PR}$ and 5 stable disease (SD) with an overall response rate of $84.4 \%$ (27/32). In 28 cases of postoperative cancer tissue specimens, a clear boundary between the cancer tissue and the breast tissue and white cut surface and little necrosis on tumor tissue were observable with the naked eye. Various degrees of cell degeneration and necrosis of the tumor cells, nuclear contraction, rupture and cytoplasmic coagulation necrosis of the cells surrounding the tumor tissue and vascular endothelial hyperplasia and blood vessel narrowing or occlusion were observed with a light microscope. In the remaining four cases, various degrees of cell degeneration were observed, but necrosis was not clear. Various degrees of nuclear contraction and fragmentation, necrosis, calcification and fibrosis were also observed in the cancer cells in the axillary lymph nodes.

BCSG1 mRNA expression. Real time RT-PCR was performed to examine the BCSG1 mRNA level in the cancer tissues of each patient prior to and following NAC (Fig. 1). The BCSG1 mRNA level in breast cancer tissues after NAC was decreased significantly compared with that before NAC (a decrease of $\sim 10 \%$ ).

Correlation of the BCSG1 level and the effect of NAC. The expression of BCSG1 protein was determined with immuno- 
Table I. Correlation of BCSG1 expression and curative effect of NAC $(n=32)$.

\begin{tabular}{|c|c|c|c|c|}
\hline \multirow{2}{*}{$\begin{array}{l}\text { Curative } \\
\text { effect } \\
\text { of NAC }\end{array}$} & \multicolumn{4}{|c|}{ BCSG1 expression } \\
\hline & $\begin{array}{c}\text { High } \\
\text { expression }\end{array}$ & $\begin{array}{c}\text { Low } \\
\text { expression }\end{array}$ & P-value & $\mathrm{r}$ \\
\hline $\mathrm{CR}+\mathrm{PR}$ & 5 & 22 & $<0.01$ & -0.584 \\
\hline SD & 4 & 1 & & \\
\hline
\end{tabular}

BCSG1, breast cancer-specific gene 1; NAC, neoadjuvant chemotherapy; CR, complete response; $\mathrm{PR}$, partial response; $\mathrm{SD}$, stable disease.
A

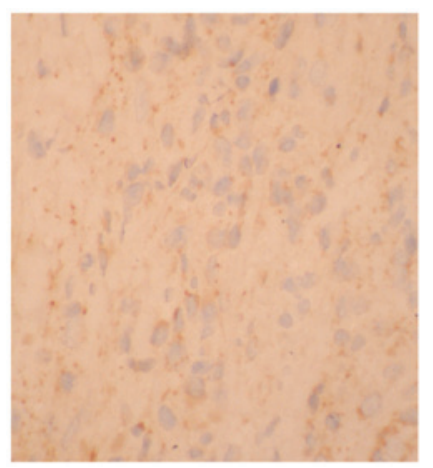

B

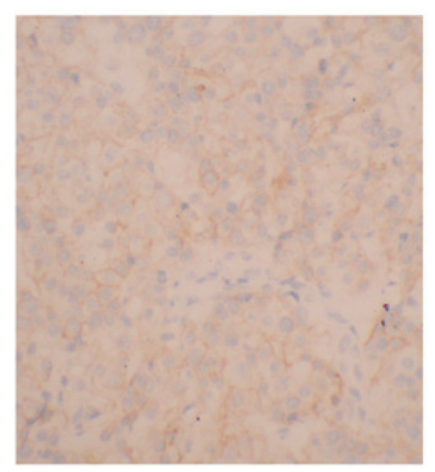

Figure 2. Immunohistochemistry for BCSG1 expression. (A) BCSG1 was markedly expressed in TNBC tissues prior to NAC (x100). (B) BCSG1 expression was lowered in TNBC tissues following NAC (x100). BCSG1, breast cancer-specific gene 1; TNBC, triple-negative breast cancer; NAC, neoadjuvant chemotherapy.

histochemistry (Fig. 2). BCSG1 protein was highly expressed in 22 patients $(68.8 \%)$ before NAC, but only in nine patients after NAC (28.1\%). The difference was significant. There was a negative correlation between the BCSG1 level and the effect of NAC ( $\mathrm{r}=-0.584, \mathrm{P}<0.01$; Table I).

\section{Discussion}

In the present study, we found that the expression of BCSG1 was decreased following NAC in TNBC patients. There was a negative correlation between the BCSG1 level and the effect of NAC in TNBC. Our results indicate that there is a correlation between the BCSG1 levels and the effect of NAC in patients with TNBC.

BCSG1, also known as SNCG ( $\gamma$-synuclein gene), was identified in 1997 by direct sequencing of cDNA gene in breast cancer (2). BCSG1 is not expressed in normal breast tissue but is highly expressed in most invasive and metastatic breast cancers. It has been shown that overexpression of BCSG1 promoted the invasion and metastasis of breast cancer cells (4). Overexpression of BCSG1 is also an event in advanced breast cancer and predicts poor clinical outcome in breast cancer $(5,6)$ These results suggest that BCSG1 is a predicator for the tumor invasion and metastasis and a target for gene therapy. Therefore, detecting BCSG1 may aid the evaluation of the invasive and metastatic ability and the prognosis of breast cancer. In our study, we found that BCSG1 was also highly expressed in TNBC patients before NAC, suggesting a potential therapy target for the TNBC. Our results showed that TNBC patients who gained more benefit from NAC had lower BCSG1 expression, indicating that BCSG1 is involved in the NAC treatment. TNBC is a subtype of breast cancer. Although TNBC is an initially chemosensitive disease, less than $25 \%$ of patients with TNBC who received standard NAC achieved pCR and the remaining patients usually have a poor prognosis (9). Several approaches have been reported to improve the NAC efficacy in previous studies, including different anthracycline-based regimens, anthracycline-taxane combinations, sequential regimens and dose-dense schedules (9). Furthermore, certain researchers have revealed other characteristics of TNBC, including overexpression of EGFR and c-KIT, increased proliferative rate through MAP kinase and Akt pathways (10) and providing some basis for the targeted therapy in those patients. Poly-ADP-ribose polymerase (PARP), a DNA-repair nuclear enzyme, has gained attention as a therapeutic target for cancer. Inhibition of PARP may improve the efficacy of certain DNA-damaging chemicals, including platinum compounds and topoisomerase inhibitors $(11,12)$. However, lack of consistency and the complexity of the analysis and interpretation of molecular classification data hindered the application of these targets in the treatment of TNBC (9). Since TNBC patients cannot benefit from target endocrine therapy and anti-HER-2 treatment, systemic chemotherapy treatment is the only one option in combination with local surgery and radiotherapy. In the present study, we showed that patients with lower BCSG1 levels after NAC gained more benefit from NAC than patients with high BCSG1 levels. Since the expression level of BCSG1 has been indicated to correlate with the effect of chemotherapy, BCSG1 would be used as a new target in NAC and a screening compound for TNBC.

The current study has certain limitations. First, the number of patients enrolled in the study was small and further research is required to support the conclusion. Second, the correlation between the BCSG1 levels and the long-term prognosis of the TNBC patients also requires further investigation in the future.

In summary, the results of the present study revealed a correlation between the BCSG1 level after NAC and the effect of NAC, indicating that BCSG1 may act as a target for chemotherapy and be used in the screening of new agents for TNBC treatment.

\section{Acknowledgements}

This study was supported by grants from the Medical Science and Technology Research Foundation of Guangdong Province (A2008605) and the Science and Technology Research Foundation of Shenzhen (no. 200802030 and no. 201101005).

\section{References}

1. Curigliano $\mathrm{G}$ and Goldhirsch A: The triple-negative subtype: new ideas for the poorest prognosis breast cancer. J Natl Cancer Inst Monogr 2011: 108-110, 2011. 
2. Ji H, Liu YE, Jia T, et al: Identification of a breast cancer-specific gene, BCSG1, by direct differential cDNA sequencing. Cancer Res 57: 759-764, 1997.

3. Wu K, Weng Z, Tao Q, et al: Stage-specific expression of breast cancer-specific gene gamma-synuclein. Cancer Epidemiol Biomarkers Prev 12: 920-925, 2003.

4. Jia T, Liu YE, Liu J and Shi YE: Stimulation of breast cancer invasion and metastasis by synuclein gamma. Cancer Res 59: 742-747, 1999.

5. Guo J, Shou C, Meng L, et al: Neuronal protein synuclein gamma predicts poor clinical outcome in breast cancer. Int J Cancer 121: 1296-1305, 2007.

6. Wu K, Quan Z, Weng Z, et al: Expression of neuronal protein synuclein gamma gene as a novel marker for breast cancer prognosis. Breast Cancer Res Treat 101: 259-267, 2007.

7. Bosch A, Eroles P, Zaragoza R, Viña JR and Lluch A: Triple-negative breast cancer: molecular features, pathogenesis, treatment and current lines of research. Cancer Treat Rev 36: 206-215, 2010
8. Mohsin SK, Zhang M, Clark GM and Craig Allred D: Maspin expression in invasive breast cancer: association with other prognostic factors. J Pathol 199: 432-435, 2003.

9. Nahleh Z: Neoadjuvant chemotherapy for 'triple negative' breast cancer: a review of current practice and future outlook. Med Oncol 27: 531-539, 2010.

10. Cleator S, Heller W and Coombes RC: Triple-negative breast cancer: therapeutic options. Lancet Oncol 8: 235-244, 2007.

11. Farmer H, McCabe N, Lord CJ, et al: Targeting the DNA repair defect in BRCA mutant cells as a therapeutic strategy. Nature 434: 917-921, 2005.

12. Ratnam K and Low JA: Current development of clinical inhibitors of poly(ADP-ribose) polymerase in oncology. Clin Cancer Res 13: $1383-1388,2007$. 